\title{
Hábitos de sueño, memoria y atención en niños escolares
}

\author{
Gisela Oropeza-Bahena ${ }^{1,2}$, Jorge D. López-Sánchez ${ }^{1,3}$ y Dora E. Granados-Ramos ${ }^{1}$ \\ ${ }^{1}$ Laboratorio de Psicobiología, Facultad de Psicología Xalapa, Universidad Veracruzana; ${ }^{2}$ Centro Estatal de Detección y Atención del Autismo, \\ CRISVer; ${ }^{3}$ Maestría en Neuroetología, Universidad Veracruzana, Xalapa. Veracruz, México
}

\begin{abstract}
Resumen
Introducción: Los hábitos de sueño son aquellas conductas que favorecen el dormir. En la etapa escolar el sueño es fundamental para el desarrollo de los niños porque durante él ocurren procesos fisiológicos de restauración del organismo. Los hábitos inadecuados de sueño (HIS) pueden ocasionar dificultades en diversos procesos cognitivos, como memoria y atención. Objetivo: Analizar la relación entre hábitos de sueño, memoria y atención en niños escolares. Métodos: Se evaluaron 52 niños (22 niñas y 30 niños), de los cuales 34 fueron del turno matutino y 18 del turno vespertino. Los padres contestaron el cuestionario de hábitos de sueño de Owens que califica los hábitos de sueño en la semana previa a la evaluación. Se consideraron las tareas de memoria de codificación y evocación de estímulos visuales-auditivos, atención auditiva y visual de la Evaluación Neuropsicológica Infantil y la tarea de memoria de trabajo de la Escala de Inteligencia Wechsler para niños. Se documentaron como factores psicosociales la cantidad de tiempo que los niños ven la televisión antes de dormir, el turno de estudio y el nivel socioeconómico. Resultados: Hubo HIS en el 80\% de los niños. En promedio, los días de escuela durmieron 9 horas y 11 horas los fines de semana. Se encontraron asociaciones negativas entre HIS con memoria de codificación auditiva, atención auditiva y ver la televisión antes de dormir. Conclusiones: Los HIS en interacción con otros factores biopsicosociales, como dormir menos horas de las recomendadas, pueden asociarse con el desempeño deficiente en los procesos de memoria y de atención en los escolares.
\end{abstract}

Palabras clave: Sueño. Memoria. Atención. Factores psicosociales. Escolares.

\section{Sleep habits, memory and attention in school children}

\section{Abstract}

Introduction: Sleep habits are behaviors that favor sleep. Sleep is fundamental for the development of children, given the physiological restoration processes of the organism that occur during it. Inadequate sleep habits can lead to difficulties in various cognitive processes, like memory and attention. Objective: To analyze the relationship between sleep habits, memory and attention processes in children of elementary school. Methods: 52 children (22 girls and 30 boys) were assessed, 34 in

\section{Correspondencia:}

Gisela Oropeza-Bahena

Laboratorio de Psicobiología

Facultad de Psicología Xalapa

Universidad Veracruzana

Av. Manantiales, $\mathrm{s} / \mathrm{n}$

Col. San Cristobal Fecha de recepción: 03-06-2018

Disponible en internet: 20-03-2019

C.P. 91097, Xalapa Enríquez, Veracruz, México Fecha de aceptación: 18-12-2018

E-mail: gisela_4893@ hotmail.com

DOI: $10.24875 /$ RMN.M19000021

Rev Mex Neuroci. 2019;20(1):42-49

www.revmexneurociencia.com

1665-5044/@ 2019. Academia Mexicana de Neurología A.C. Publicado por Permanyer México. Este es un artículo Open Access bajo la licencia CC BY-NC-ND (http://creativecommons.org/licenses/by-nc-nd/4.0/). 
the morning and 18 in the afternoon shitt. Parents answered the Children's sleep habits questionnaire, which rates sleep habits of the week before the assessment. Auditory and visual memory and attention were measured with the Evaluación Neuropsicológica Infantil and working memory was measured with the Wechsler Intelligence Scale for Children. The amount of time children watched television before bed time, the study shitt, and the socioeconomic level were documented as psychosocial factors. Results: There were inadequate sleep habits in $80 \%$ of children; on average, during school days children slept $9 \mathrm{~h}$ and $11 \mathrm{~h}$ on weekends. Negative correlations were found between inadequate sleep habits and auditory encoding, auditory attention and watching television before bed time. Conclusions: Inadequate sleep habits in interaction with other biopsychosocial factors, such as sleeping less than recommended hours, may be associated with poor performance in memory and attention processes in school children.

Key words: Sleep. Memory. Attention. Psychosocial factors. School children.

\section{Introducción}

Los hábitos de sueño saludables favorecen el dormir adecuadamente. Incluyen diversos cambios en la rutina de sueño, desde el ambiente en donde se duerme hasta las actividades que se realizan antes de dormir; estas conductas también se conocen como medidas de higiene del sueño. Es importante señalar que el sueño es una conducta aprendida que puede reaprenderse para dormir bien ${ }^{1}$. Con la adecuada educación por parte de los padres y los cuidadores desde el nacimiento del niño, la mayoría de los problemas y trastornos del sueño podrían prevenirse, lo cual favorece la calidad de vida del niño $0^{2-3}$.

El sueño en la infancia es fundamental debido a que mientras se duerme ocurren diversos procesos de maduración y desarrollo del sistema nervioso, como la liberación de la hormona del crecimiento, la consolidación de la memoria, la regulación metabólica y el restablecimiento de la energía ${ }^{4-5}$. Por ello, la presencia de problemas de sueño puede dar lugar a alteraciones en el desarrollo, deficiencias en la memoria, en la atención y en el aprendizaje ${ }^{6}$.

Como ya se mencionó, no contar con higiene de sueño puede generar problemas en el buen dormir y si no se da atención oportuna a dichos problemas pueden llegar a convertirse en trastornos de sueño ${ }^{7}$. Se considera que uno de cada tres niños puede presentar problemas al dormir, además es común que estos pasen desapercibido para los padres y los pediatras $^{4,8}$. La alta prevalencia de trastornos del sueño en niños es un tema importante en salud por su alta prevalencia y sus efectos negativos en el desarrollo ${ }^{3}$. En México se estima que entre el 10 y el $40 \%$ de los niños en edad preescolar y escolar presentan algún trastorno de sueño ${ }^{4}$. Por lo anterior, el objetivo del estudio fue analizar la relación entre los hábitos de sueño, la memoria y la atención en los niños escolares.

\section{Métodos}

Se realizó un estudio de tipo observacional, transversal, descriptivo y comparativo con 52 niños de educación primaria de primero a sexto grado, 34 niños del turno matutino y 18 del vespertino de escuelas públicas de la ciudad de Xalapa, Veracruz. Se llevó a cabo en un periodo de 8 meses (junio 2015-enero 2016). Como criterios de inclusión se consideró que todos los niños tuvieran de 6 a 12 años de edad y un coeficiente intelectual $(\mathrm{Cl})$ de 80 a 109, valorado con la Escala Wechsler de Inteligencia para niños mexicanos (WISC-IV). No se incluyó a los niños que no cumplieron con estos criterios o presentaron algún trastorno del neurodesarrollo como Trastorno por déficit de Atención e Hiperactividad (TDAH), autismo o problemas de salud. Los padres firmaron una carta de consentimiento informado donde se les comunicaron los objetivos de la investigación y las pruebas que se realizarían a sus hijos, y los niños firmaron una carta de asentimiento.

Se consideraron para esta investigación las siguientes variables: hábitos de sueño, memoria de codificación auditiva (MCA), memoria de codificación visual (MCV), memoria de evocación auditiva (MEA), memoria de evocación visual (MEV), memoria de trabajo (MT) atención auditiva (AA), atención visual (AV), turno de estudio, sexo y factores psicosociales.

Para medir los hábitos de sueño los padres contestaron el Cuestionario de hábitos de sueño de Owens (CHSO) ${ }^{9}$ en su versión en español, el cual tiene consistencia interna de 0.68 a 0.78 , sensibilidad de 0.80 y especificidad de 0.72 . El CHSO es una escala tipo Likert de 33 preguntas que permite conocer los hábitos de sueño de los niños durante la semana anterior a la evaluación. Está diseñado para valorar a niños de 4 a 12 años de edad; evalúa el sueño con ocho subescalas diferentes: resistencia a acostarse (RA), retraso de inicio del sueño, duración de sueño, ansiedad al dormir, despertares nocturnos, parasomnias, problemas respiratorios y somnolencia diurna. Se registra 


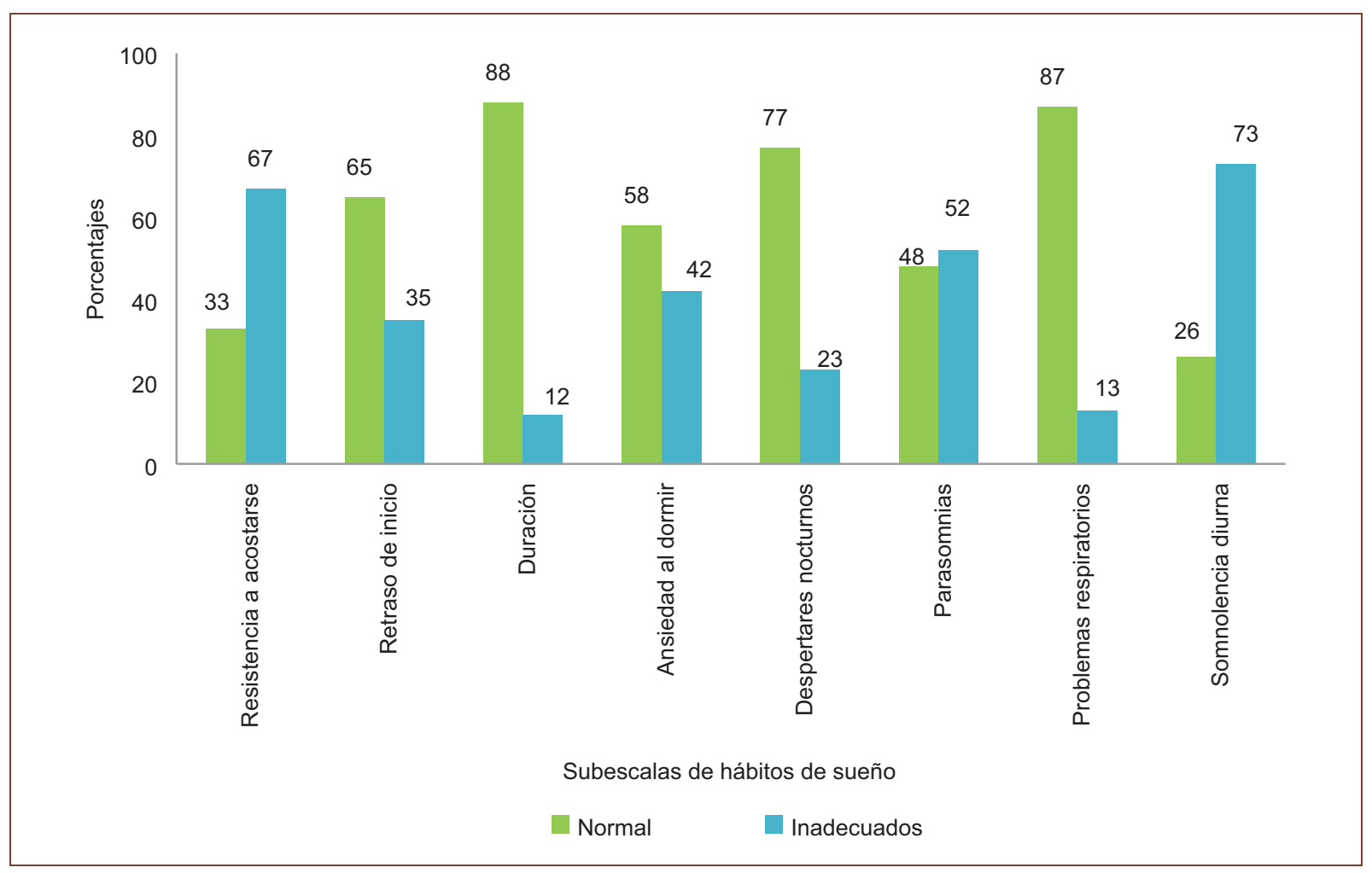

Figura 1. Subescalas del cuestionario de hábitos de sueño de Owens.

la frecuencia con la que el niño realiza las conductas enlistadas en una semana y se clasifica en: habitualmente (5 veces por semana), con valor de 3 puntos; a veces (2 a 4 veces por semana), con valor de 2 puntos, o raro (0 a 1 vez por semana), con valor de 1 punto. Para obtener el puntaje final se sumaron los resultados de cada reactivo. Los puntajes mayores de 41 puntos se consideraron como presencia de hábitos inadecuados de sueño (HIS) en una o varias de las subescalas.

Para medir el $\mathrm{Cl}$ y la MT se utilizó el WISC- IV ${ }^{10}$ estandarizado para población mexicana. Los puntajes se clasificaron como: > 130, muy superior; $120-129$, superior; 110-119, promedio alto; 90-109, promedio; 80-89, promedio bajo; 70-79, límite, y $<69$, muy bajo. Se incluyeron para esta investigación los participantes que obtuvieron puntajes de 80 a 109.

Para medir la MCA, la MCV, la MEA, la MEV, la AA y la AV se realizó la Evaluación Neuropsicológica Infantil $(E N I)^{11}$, la cual examina el desarrollo neuropsicológico de la población infantil de habla hispana en edades de 5 a 16 años. Se califica en rangos de: $>75$, por arriba del promedio; $26-75$, promedio; $11-25$, promedio bajo; 3-10, bajo, y $\leq 2$, extremadamente bajo. Para esta investigación se consideraron los puntajes $\geq 26$ como promedio $\mathrm{y}<26$ bajos.
En los factores psicosociales se documentaron: el turno de estudio, la cantidad de horas que ven la televisión los niños antes de dormir y el nivel socioeconómico de los padres, el cual se obtuvo con el ingreso mensual de acuerdo a la clasificación de la Asociación Mexicana de Agencias de Investigación de Mercado: pobreza extrema ( $\$ 0$ a $\$ 2,699)$, clase pobre $(\$ 2,700$ a $\$ 6,799)$, clase media baja $(\$ 6,800$ a $\$ 11,599)$, clase media ( $\$ 11,600$ a $\$ 34,999)$, media alta $(\$ 35,000$ a $\$ 84,999)$ y clase rica (mayor a $\$ 85,000)^{12}$.

Se realizó el análisis estadístico con el programa SPSS $^{\circledR}$ v20 para Windows, se obtuvieron estadísticos descriptivos. Se emplearon pruebas no paramétricas debido a que la distribución de los datos no cumplió los criterios de normalidad. Se analizó la correlación entre las variables: hábitos de sueño, memoria, atención, horas de sueño y factores psicosociales. Se realizó la prueba de rangos de Wilcoxon para analizar si existían diferencias entre los turnos de estudio (matutino y vespertino).

\section{Resultados}

La muestra estuvo compuesta por 10 niños de primero, 12 de segundo, 11 de tercero, 11 de cuarto, 5 de quinto y 3 de sexto grado de educación primaria. La 


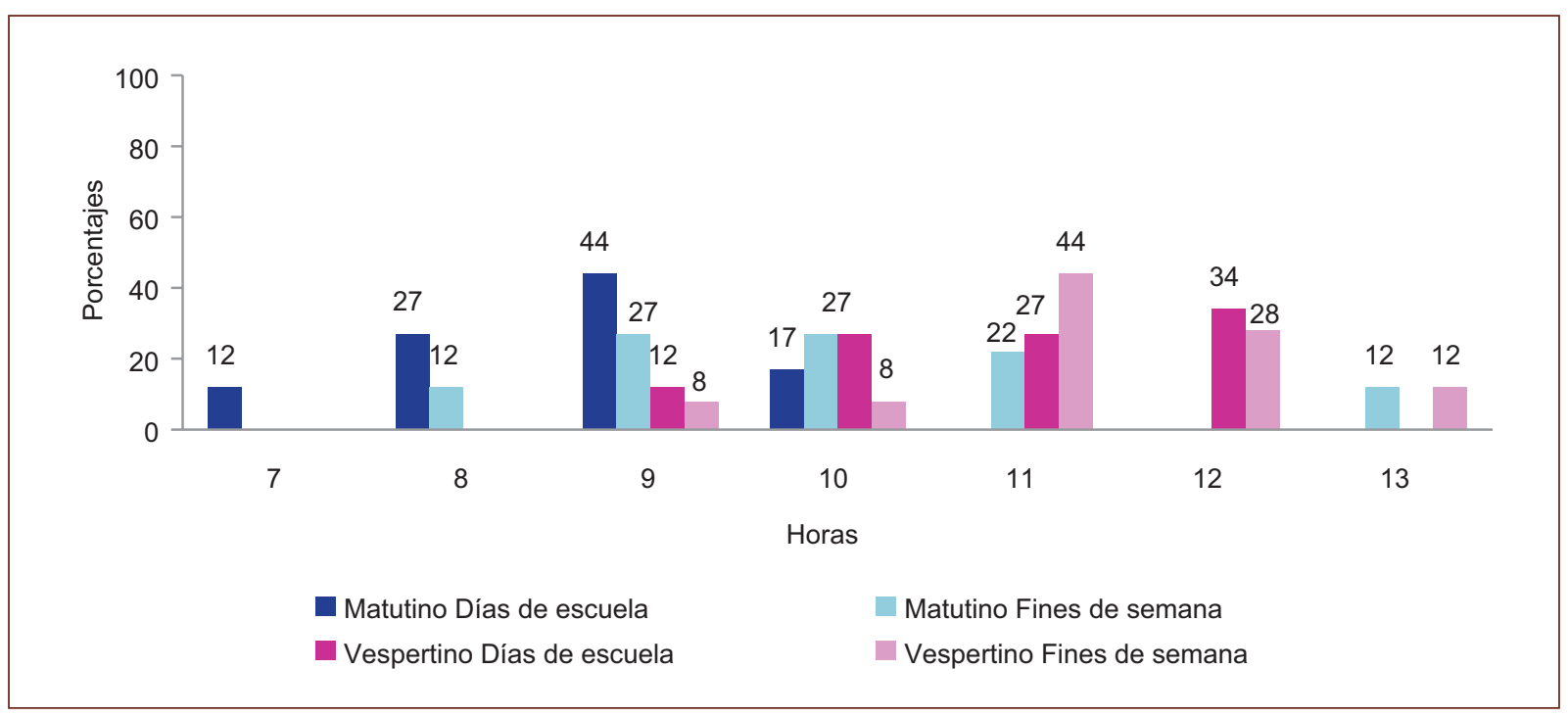

Figura 2. Horas de sueño de los niños escolares.

edad promedio fue de 8 años y 5 meses con desviación estándar (DE): 1.2. El promedio grupal de $\mathrm{Cl}$ fue de 98, con DE: 9.

De acuerdo al instrumento $\mathrm{CHSO}$, se encontró que el $80 \%$ de los niños presentó HIS, correspondiendo los mayores porcentajes a las subescalas de somnolencia diurna, RA y parasomnias (Fig. 1).

El mínimo de horas que durmieron los niños fue de 7 y el máximo de 13; de lunes a viernes durmieron en promedio 9 horas y los fines de semana 11 horas. En el turno matutino el $83 \%$ de los niños durmieron menos de 9 horas entre semana y el $17 \%$ más de 10 horas. Los fines de semana el $39 \%$ de los niños del turno matutino durmieron menos de 9 horas y el $61 \%$ más de 10 horas. En el turno vespertino el $12 \%$ de los niños durmieron menos de 9 horas entre semana y el $88 \%$ más de 10 horas. Los fines de semana el $8 \%$ de los niños del turno vespertino durmieron menos de 9 horas y el $92 \%$ más de 10 horas (Fig. 2).

En los niños evaluados se observó que en las variables de memoria y atención predominaron los puntajes promedio (26-75), siendo mayor la proporción de puntajes promedio para la MCV con un $71 \%$ y para la AA con un $82 \%$ (Fig. 3).

Al comparar los hábitos de sueño por sexo se encontró que el $73 \%$ de las niñas y el $90 \%$ de los niños presentaron hábitos inadecuados. En las pruebas de memoria y atención la mayoría de los niños evaluados obtuvieron puntajes promedio: el $67 \%$ de las niñas y el $70 \%$ de los niños en MCV, el $59 \%$ de las niñas y el $67 \%$ de los niños en MEA, el $68 \%$ de las niñas y el $70 \%$ de
Ios niños en MCA y el $68 \%$ de las niñas y el $67 \%$ de Ios niños en MEV. En atención, las niñas tuvieron mayor porcentaje en $\mathrm{AA}(91 \%)$ que los niños $(76 \%)$ y en $\mathrm{AV}$ los niños obtuvieron mayor porcentaje $(70 \%)$ que las niñas (64\%).

Al comparar los hábitos de sueño por turno de estudio, se encontró que mayor porcentaje de los niños del turno matutino presentó somnolencia diurna (72\%), RA (50\%) y despertares nocturnos (39\%); por otro lado, en el turno vespertino se observó RA (83\%), retraso de inicio de sueño $(66 \%)$ y somnolencia diurna (45\%) (Fig. 4). Los niños del turno vespertino obtuvieron puntajes mayores en MCV, MEA, $M E V, A A$ y $A V$, y los niños del turno matutino en MCA (Fig. 5).

En los factores psicosociales se encontró que el $80 \%$ de los niños veía la televisión antes de dormir y el 57\% la veía una hora en promedio. El nivel socioeconómico se distribuyó de la siguiente manera: $4 \%$ de clase media, $56 \%$ de clase media baja, $29 \%$ pobre y $11 \%$ en pobreza extrema.

Se encontraron correlaciones negativas significativas entre las variables: HIS con MCA, MT con duración de sueño (DS) y AA con RA. Se encontraron correlaciones positivas significativas entre las variables: horas de televisión (HTV) antes de dormir con HIS y AA con la duración de sueño los fines de semana (DSF) (Tabla 1).

No se encontraron diferencias significativas al comparar por sexo y por nivel socioeconómico las variables de hábitos de sueño, memoria y atención. Al comparar 


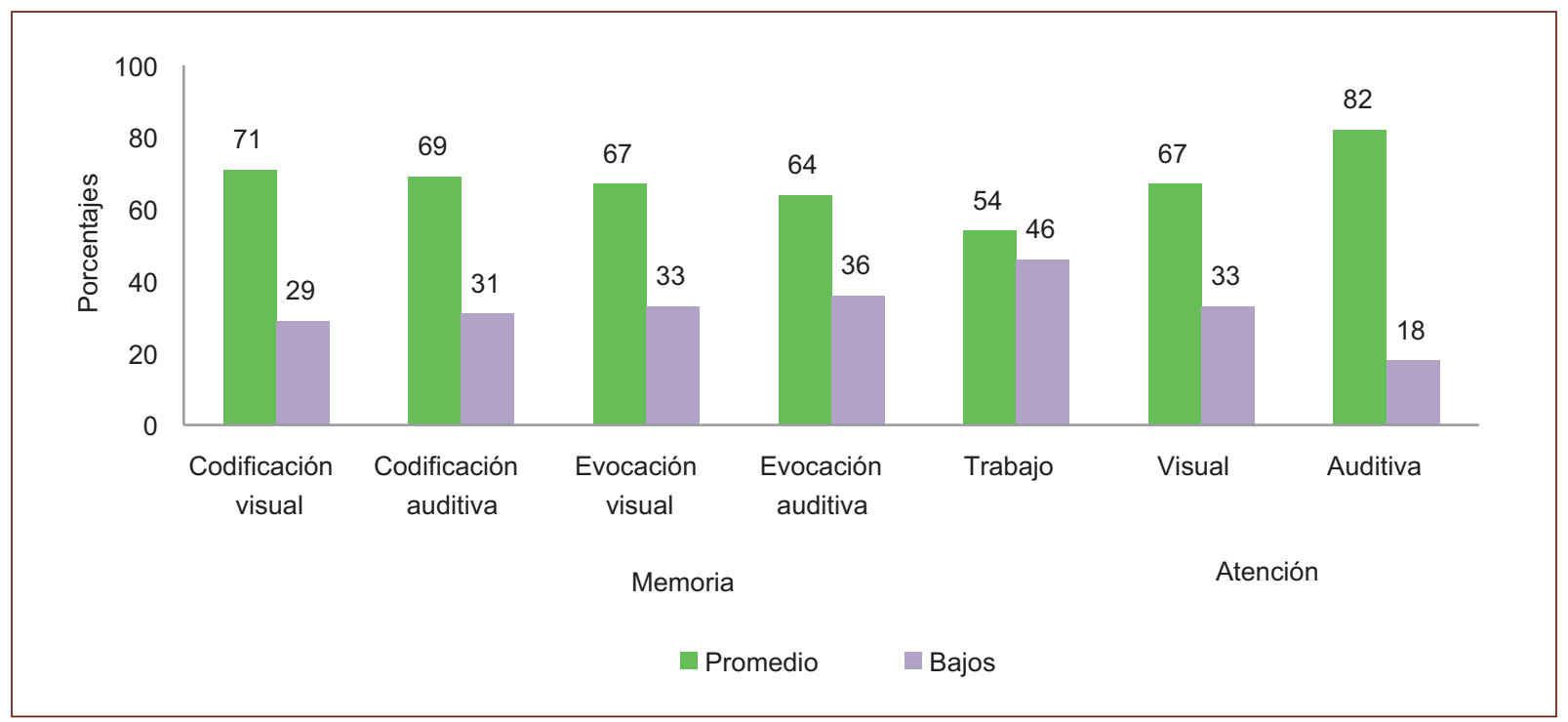

Figura 3. Resultados de memoria y atención.

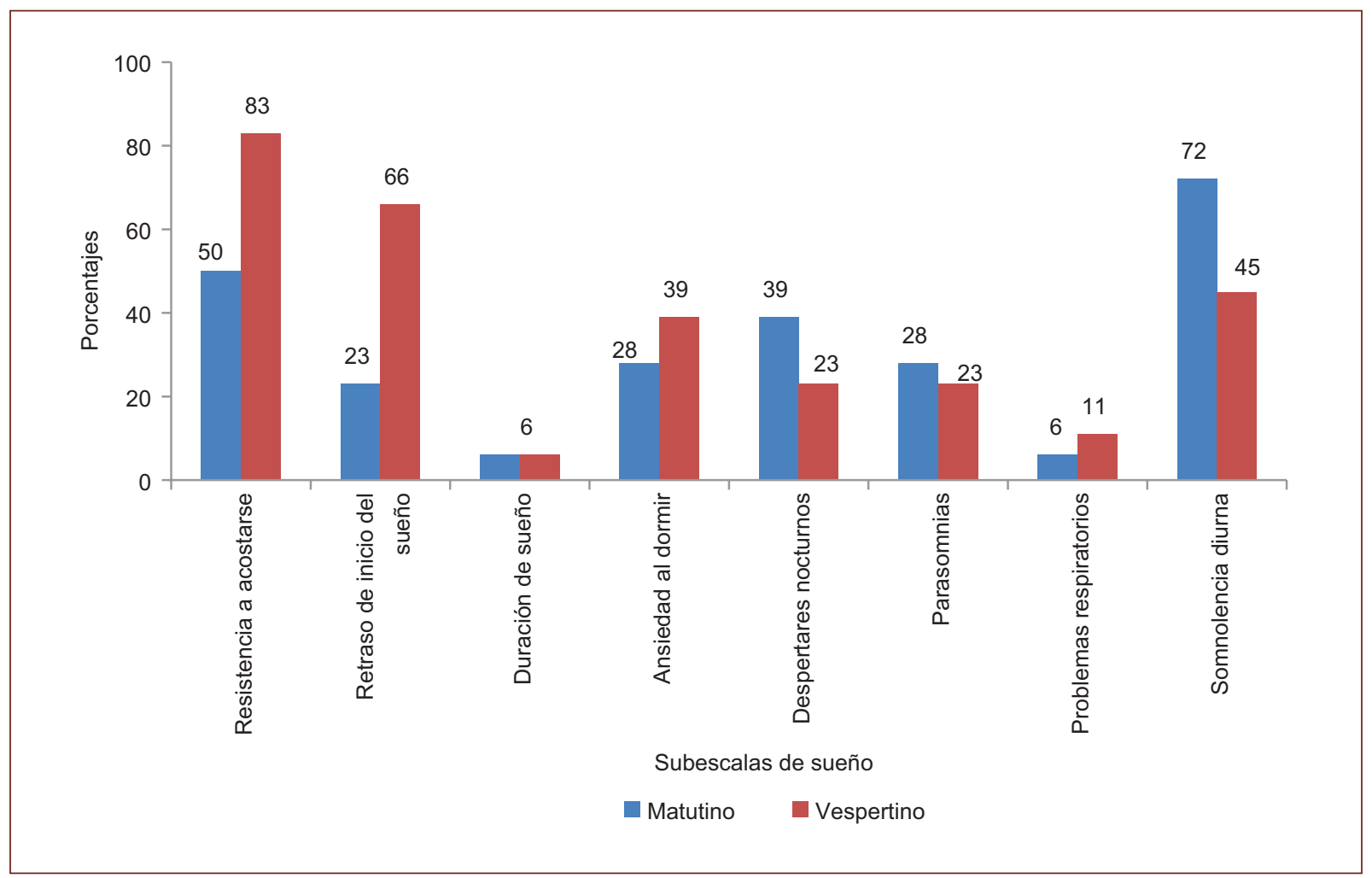

Figura 4. Hábitos inadecuados de sueño por turno de estudio.

por turno de estudio (matutino y vespertino) se encontraron diferencias significativas: los niños del turno vespertino tuvieron puntajes mayores en MCV (estadístico de la prueba de Wilcoxon [W]: 186; $p=0.020$ ) (matutino, mediana [Mdn]: 26, vespertino Mdn: 37), MEV (W: 177; $p=0.012$ ) (matutino Mdn: 37 , vespertino Mdn: 50) y AV (W: $179.5 ; p=0.014$ ) (matutino Mdn: 26 , vespertino Mdn: 37). 


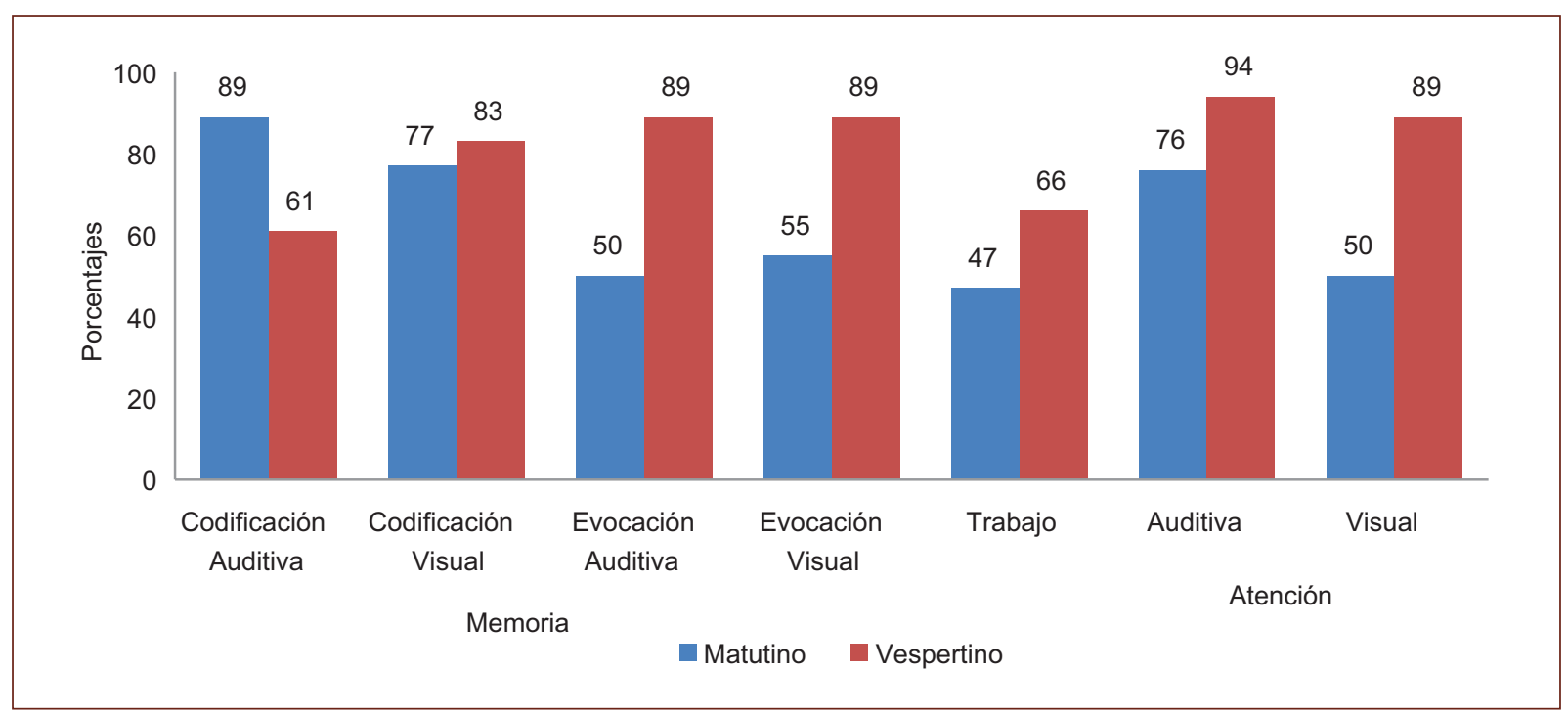

Figura 5. Calificaciones promedio de los procesos neuropsicológicos valorados por turno de estudio.

Tabla 1. Correlaciones Rho de Spearman

\begin{tabular}{|l|l|l|l|l|l|l|l|l|}
\hline & MCA & MT & AA & HIS & RA & DS & DSF & HTV \\
\hline MCA & 1.000 & & & & & \\
\hline MT & $0.292^{*}$ & 1.00 & & & & \\
\hline AA & $0.290^{*}$ & 0.212 & 1.00 & & & \\
\hline HIS & $-0.353^{*}$ & -0.122 & $-0.377^{\dagger}$ & 1.00 & & \\
\hline RA & -0.250 & -0.073 & $-0.401^{\dagger}$ & $0.552^{\dagger}$ & 1.00 & & \\
\hline DS & -0.269 & $-0.292^{*}$ & -0.079 & $0.449^{\dagger}$ & 0.252 & 1.00 & \\
\hline DSF & 0.157 & -0.052 & $0.310^{*}$ & -0.263 & -0.076 & $0.543^{\dagger}$ & 1.00 \\
\hline HTV & -0.170 & -0.166 & -0.070 & $0.359^{\dagger}$ & 0.192 & -0.027 & 0.049 \\
\hline
\end{tabular}

MCA: memoria de codificación auditiva; MT: memoria de trabajo; HIS: hábitos de sueño inadecuados; RA: resistencia a acostarse; DS: duración de sueño; DSF: duración de sueño los fines de semana; HTV: horas de ver televisión.

${ }^{*} p<0.05$

${ }^{\dagger} \mathrm{p}<0.01$.

\section{Discusión}

Es importante estudiar los hábitos de sueño en niños debido a su impacto en el desarrollo. El sueño adecuado conlleva a mejor rendimiento en el aprendizaje, la capacidad de focalizar la atención y la consolidación de la memoria, mejora las estrategias cognitivas y la salud de los niños escolares ${ }^{13-14}$. En cambio, la privación de sueño, dormir menos horas de las recomendadas, la somnolencia diurna y los HIS pueden afectar el rendimiento diurno, el desempeño académico y el desarrollo de las competencias básicas ${ }^{14}$.

El objetivo de la investigación se cumplió, ya que se analizaron las variables de hábitos de sueño, memoria y atención en niños escolares. Las correlaciones negativas encontradas entre los HIS con los puntajes de memoria y atención indican que ante la presencia de mayor cantidad de hábitos inadecuados, fueron menores los puntajes de memoria y atención, esto puede atribuirse a que los hábitos inadecuados ocasionan mala calidad de sueño, lo cual conlleva complicaciones como: dificultades para sostener la atención por periodos prolongados de tiempo e inhibir estímulos distractores, así como la disminución de la capacidad de memoria y del aprendizaje ${ }^{15-16}$.

El problema de sueño más reportado fue la somnolencia diurna, esto se debe a que el número de horas que durmieron los niños fue menor que las 10 a 11 
horas recomendadas en la edad escolar ${ }^{2}$. La reducción de horas de sueño empeora el rendimiento neurocognitivo, psicomotor y académico ${ }^{15}$, además se ha encontrado que a mayor duración del sueño mejora la retención de la información ${ }^{16,17}$.

Respecto a la asociación entre la cantidad de horas de ver televisión y el mayor número de problemas de sueño observados en los niños, algunos estudios ${ }^{18-22}$ concluyeron que ver durante más tiempo la televisión repercute en el sueño, ocasionando insomnio y menos horas de sueño. Por otro lado, también se ha reportado que ver menos tiempo la televisión mejora la calidad de sueño. Lo anterior se explica porque la televisión, o cualquier otro dispositivo electrónico, emiten luz azul $(450 \mathrm{~nm})$ que provoca activación fisiológica. Dicha luz disminuye la producción de melatonina, incrementa la temperatura corporal, dificulta el inicio del sueño, genera retrasos del ritmo circadiano y menor DS.

Los alumnos del turno vespertino tuvieron mejor desempeño en las tareas de memoria y atención en comparación con los del turno matutino, una de las variables que pudo influir fue que los alumnos del turno vespertino durmieron más horas. Se ha descrito que dormir las horas recomendadas para la edad favorece la consolidación de los aprendizajes y mejora la ejecución en tareas motoras, visuales y verbales ${ }^{16}$.

Los cuestionarios de sueño se emplean como pruebas de tamizaje en los niños, debido a su practicidad, bajos costos y aplicación rápida. Si bien no pueden sustituir a técnicas como la polisomnografía y la actigrafía, se han validado como herramientas adecuadas para describir el sueño e identificar trastornos del sueño?.

Es importante conocer los hábitos de sueño de los niños para generar estrategias que permitan corregir los hábitos inadecuados, como el uso de dispositivos electrónicos y el consumo de bebidas estimulantes (café, refrescos, chocolate o té negro), ya que son conductas que pueden modificarse ${ }^{13}$. Se debe educar a los padres sobre la importancia del sueño en los niños y cómo favorecer hábitos adecuados; establecer una rutina dos horas antes de dormir que ayude a relajar al niño, como cenar ligero, cepillarse los dientes, ponerse la pijama, leer textos cortos, apagar las luces y acostarse. Es importante recordar que, además del descanso, durante el día las actividades de juego, alimentación, tareas escolares y de casa deben estar organizadas.

Si se establecen hábitos de sueño adecuados en etapas tempranas es probable que permanezcan hasta la edad adulta, por ello es importante dar a conocer la relevancia del sueño en el desarrollo de los niños y promover los hábitos saludables con los padres, maestros y especialistas de la salud, ya que varios de los problemas de sueño pasan desapercibidos o no se les da importancia?.

Esta investigación tiene como limitaciones ser de diseño transversal, lo que no nos permite asegurar que los hábitos adecuados permanezcan estables a lo largo del año, por lo que un estudio longitudinal permitiría realizar diferentes mediciones a lo largo del ciclo escolar. Por otro lado se midió con un instrumento contestado por los padres, por lo que sería relevante diseñar instrumentos que valoren la percepción de los niños para complementar la información. Finalmente, no se consideró la influencia de variables como: características de la dieta, clima, cambios de horario, hábitos de sueño de los padres y contexto donde viven. Estas variables permitirían explicar con detalle los factores asociados a los hábitos adecuados e inadecuados de sueño en los niños escolares, encontrar la explicación del mejor desempeño en los niños del turno vespertino y generar estrategias específicas.

\section{Conclusión}

Los HIS como dormir menos horas de lo recomendado, resistencia a ir a la cama y el uso de dispositivos electrónicos antes de dormir pueden generar somnolencia diurna, afectar la cantidad y calidad del sueño, afectar los procesos de memoria, atención y desempeño en los escolares.

Habrá que considerar otras variables biopsicosociales que contribuyan a los hábitos de sueño y a los procesos cognoscitivos adecuados.

\section{Declaración de conflictos de interés}

Los autores declaran que no existen conflictos de interés relevantes para este artículo de revisión.

\section{Fuentes de financiamiento}

No existió una fuente de financiamiento particular para este informe científico.

\section{Bibliografía}

1. Carrillo-Mora P, Ramírez-Peris J, Magaña-Vázquez K. Neurobiología del sueño y su importancia: antología para el estudiante universitario. Rev Fac Med (Méx). 2013;56:5-15.

2. Cacho Y. Trastornos de sueño, un problema importante en México [Internet]. México: Conacyt agencia informativa; 2016 [consultado el 20 de enero de 2017]. Disponible en: http://www.conacytprensa.mx/index.php/ ciencia/salud/5055-trastornos-de-sueno-un-problema-importante-en-mexico). 
3. Masalán $P$, Sequieda J, Ortiz M. Sueño en escolares y adolescentes, su importancia y promoción a través de programas educativos. Rev Chil Pediatr. 2013;84:554-64.

4. Vicoso J. El sueño y sus trastornos. En: Barragán E, editor. Neurodesarrollo variantes, perspectivas y trastornos. México: Ediciones Graficas Deseret; 2011.

5. Hernández J, García M, Antona M, Sanz A, Cordero P. Hábitos y trastornos del sueño en adolescentes. Rev Esp Patol Torac. 2015;27(4):220-30.

6. García F, Nachón F, Hernández G. Ritmos biológicos. En: Coria G, editor. Neurofisiología de la conducta, cerebro y comportamiento. México: Universidad Veracruzana; 2012.

7. Cruz Navarro IJ. "La vida es sueño.": lo que Calderón de la Barca no sabía sobre el sueño infantil Introducción. En: AEPa, ed. Curso de actualización Pediatría. Madrid: Lúa Ediciones 3.0; 2017.

8. Pin G, Morell M y Mompo L. Higiene del sueño y melatonina. Curso de Actualización Pediatría. Madrid: Exlibris Ediciones;2014;133-4.

9. Owens J, Spirito A, McGuinn M. The Children's Sleep Habits Questionnaire (CSHQ): psychometric properties of a survey instrument for schoolaged children. Sleep. 2000;23(8):1-9.

10. Wechsler D. WISC-IV. Escala Wechsler de Inteligencia para Niños-IV. México: Manual Moderno; 2007.

11. Matute E, Rosselli M, Ardila A, Ostrosky-Solís F. Evaluación Neuropsicológica Infantil (ENI): Manual de aplicación. México: Manual Moderno; 2007.

12. ¿Cuánto ganan al mes los hogares en México por nivel socioeconómico? [Internet]. México: Amedirh, Asociación Mexicana en Dirección de Recursos Humanos A.C.; 2014 [fecha de acceso: 4 mayo, 2017] Disponible en: http://www.amedirh.com.mx/publicaciones/noticias/item/cuanto-ganan-al-mes-los-hogares-en-mexico-por-nivel-socioeconomico.
13. Manonellas S, Ortiz R, Agudo J, Vilaró A, Aparicio M, Pretel L. Hábitos del sueño en niños de 8 a 12 años escolarizados en el barrio "La Mina". Metas enferm. 2016;19(10):6-14.

14. Cladellas R, Chamarro A, Badía MM, Oberst U, Carbonell X. Efectos de las horas y los hábitos de sueño en el rendimiento académico de niños de 6 y 7 años: un estudio preliminar. C\&E. Cult. educ. 2011;23(1):119-28.

15. Arrona-Palacio A, García A, Valdez P. Sleep-wake habits and circadian preference in Mexican secondary school. Sleep Medicine. 2015;16:1259-64.

16. Corsi M. ¿Qué le pasa al cerebro cuando no duerme?. Rev Med UV. 2008;8(1):53-6.

17. Guzmán E. Sueño, sueños y aprendizaje hacia una neurofisiología de la cognición. Acta Med Colomb. 1992;17(4):258-65.

18. Custers $K$, van den Bulck J. Television viewing, internet use, and self-reported bedtime and rise time in adults: implications for sleep hygiene recommendations from an exploratory cross-sectional study. Behav Sleep Med. 2012;10(2):96-105.

19. Nesdal I, Tinnesand L, Straume S, Bjorvatn B, Pallesen S. The association between use of electronic media in bed before going to sleep and insomnia symptoms, daytime sleepiness, morningness, and chronotype. Behav Sleep Med. 2013;12(5):343-57.

20. Marinelli M, Sunyer J, Alvarez-Pedrerol M, Iñiguez C, Torrent M, Julvez J. Hours of television viewing and sleep duration in children: a multicenter birth cohort study. JAMA Pediatric. 2014;168(5):458-64.

21. Lillo J, Álvaro L, Moreira H, Durán M. Luz y ritmos circadianos: efectos en la salud y en el desempeño. Ansiedad Estrés. 2010;16(2-3):259-69.

22. Sekine M, Chen X, Hamanishi S, Wang H, YamagamiT, Kagamimori S. The validity of sleeping hours of healthy young children as reported by their parents. Epidemiol. 2002;12(3):237-4. 